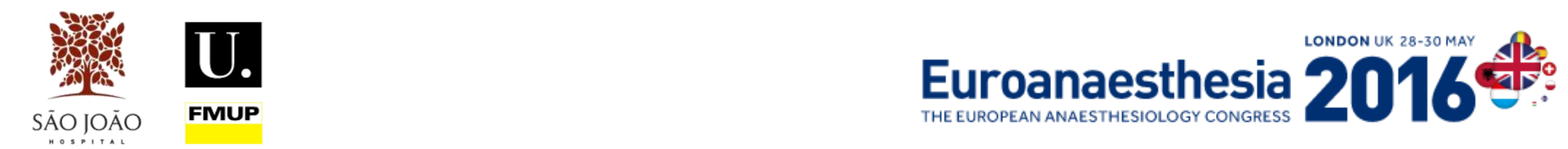

\title{
Myocardial Infarction after Endovascular Repair of Abdominal Aortic Aneurysms
}

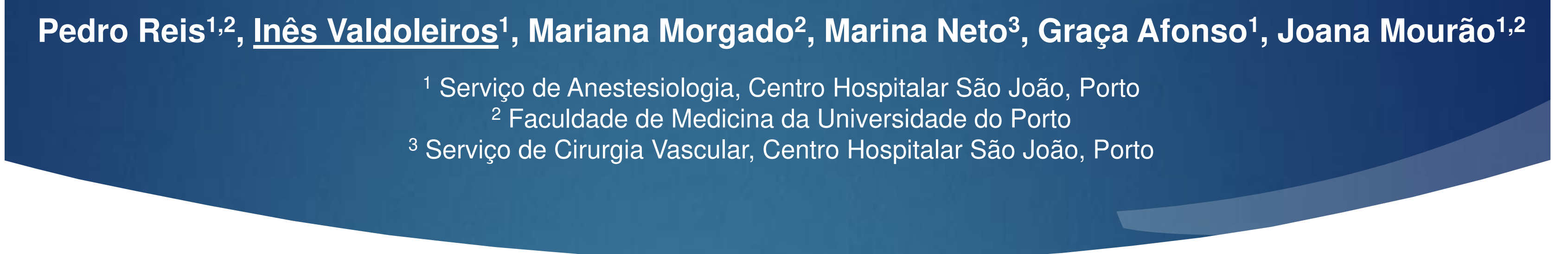

- Background and Goal of Study

- The endovascular repair of abdominal aortic aneurysms (EVAR) has expanded with encouraging results, contributing to a change in the practice of vascular surgery and anaesthesia. Vascular surgery patients have many comorbidities associated with myocardial infarction (MI), frequently asymptomatic in the perioperative period.

- Aim of study: to evaluate the incidence, predictors and outcomes of MI after EVAR.

\section{- Materials ANd Methods}

- Retrospective study of patients undergoing TEVAR from 2006 to 2013.

- Patients' demographics and perioperative data were collected.

- Excluded patients without sufficient data available on clinical records.

- MI: troponin rise superior to $0,034 \mathrm{ng} / \mathrm{ml}$ in the first 72 hours postoperatively.

- Acute Kidney Injury (AKI): rise of $0.3 \mathrm{mg} / \mathrm{dl}$ in postoperative serum creatinine.

- Statistical analysis: descriptive analysis, Student's T test, Mann-Whitney test, Fischer's exact test, Chi-square test. Univariate logistic regression with calculation of an Odds Ratio (OR) and its 95\% Confidence Interval (Cl).

Table 2. Multivariate analysis of predictors of MI.

\begin{tabular}{c|c|c|c|c|}
\hline VARIABLE & $\begin{array}{c}\text { Simple OR } \\
{[\text { IC 95\%] }}\end{array}$ & $p$ & $\begin{array}{c}\text { Adjusted OR } \\
{[\text { CI 95\%] }}\end{array}$ & $p$ \\
\hline General anaesthesia & $8.5[1.30-55.3]$ & $\mathbf{0 . 0 2 6}$ & - & - \\
\hline Postoperative AKI & $24.4[2.5-238.7]$ & $\mathbf{0 . 0 0 6}$ & $24.4[2.5-238.7]$ & $\mathbf{0 . 0 0 6}$ \\
\hline
\end{tabular}

\section{Conclusions}

- European Society of Cardiology Guidelines consider EVAR as an intermediate risk surgery with $1-5 \%$ expected $\mathrm{MI}$ as we observed.

- General anaesthesia and postoperative AKI increased the risk of MI.
- Results And Discussion

- Included 98 patients. $\Rightarrow$ Incidence of MI: $5 \%$

\begin{tabular}{|c|c|c|c|}
\hline VARIABLE & No MI (n=93) & $M I(n=5)$ & $p$ \\
\hline Age $^{1}$ & $75 \pm 6.5$ & $73 \pm 11.2$ & $0.698^{b}$ \\
\hline Male gender ${ }^{2}$ & $88(95)$ & $5(100)$ & $0.595^{\mathrm{a}}$ \\
\hline ASA II/ III' & $82(88)$ & $5(100)$ & \multirow{2}{*}{$>0.999 c$} \\
\hline ASA IV/ V 2 & $11(12)$ & $0(0)$ & \\
\hline Arterial hypertension ${ }^{2}$ & $12(13)$ & $0(0)$ & $>0.999^{a}$ \\
\hline Dyslipidemia $^{2}$ & $83(89)$ & $5(100)$ & $>0.999^{a}$ \\
\hline Ischemic heart disease ${ }^{2}$ & $22(24)$ & $2(40)$ & $0.593^{a}$ \\
\hline Obesity $^{2}$ & $37(40)$ & $3(60)$ & $0.396^{\mathrm{a}}$ \\
\hline Chronic obstructive pulmonary disease ${ }^{2}$ & $61(66)$ & $2(40)$ & $0.345^{\mathrm{a}}$ \\
\hline Heart failure $^{2}$ & $20(22$ & $0(0)$ & $0.580^{\mathrm{a}}$ \\
\hline Diabetes mellitus $^{2}$ & $10(11)$ & $1(20)$ & $0.456^{\mathrm{a}}$ \\
\hline Cerebrovascular disease ${ }^{2}$ & $9(97)$ & $0(0)$ & $>0.999^{a}$ \\
\hline Chronic kidney disease ${ }^{2}$ & $23(25)$ & $0(0)$ & $0.585^{\mathrm{a}}$ \\
\hline Peripheral artery disease ${ }^{2}$ & $27(29)$ & $0(0)$ & $0.318^{a}$ \\
\hline Diuretic $^{2}$ & $32(34)$ & $3(60)$ & $0.345^{\mathrm{a}}$ \\
\hline$\beta$-Blocker ${ }^{2}$ & $28(30)$ & $3(60)$ & $0.322^{\mathrm{a}}$ \\
\hline Statin $^{2}$ & $53(57)$ & $5(100)$ & $0.077^{a}$ \\
\hline Anticoagulation ${ }^{2}$ & $8(9)$ & $1(20)$ & $0.389^{a}$ \\
\hline Digoxin² & $2(2)$ & $0(0)$ & $>0.999^{a}$ \\
\hline Antiaggregation ${ }^{2}$ & $51(55)$ & $5(100)$ & $0.069^{a}$ \\
\hline Preoperative haemoglobin $(\mathrm{g} / \mathrm{d} \mathbf{l})^{1}$ & $13.3 \pm 1.9$ & $13.9 \pm 2.1$ & $0.449^{b}$ \\
\hline Preoperative haematocrit $(\%)^{3}$ & $40.3[36.4-43.6]$ & $42.4[36.0-45.9]$ & $0.607^{c}$ \\
\hline Preoperative creatinine $(\mathrm{mg} / \mathrm{dl})^{3}$ & $1.16[0.99-1.50]$ & $1.36[1.22-1.43]$ & $0.385^{c}$ \\
\hline Preoperative creatinine $>1.2(\mathrm{mg} / \mathrm{dl})^{2}$ & $33(42)$ & $4(80)$ & $0.164^{c}$ \\
\hline Preoperative urea $(\mathrm{mg} / \mathrm{dl})^{3}$ & $48.5[38-61]$ & $60[46-64.5]$ & $0.298^{c}$ \\
\hline Aneurysm diameter $(\mathrm{cm})^{3}$ & $6.0[5.3-7.0]$ & $6.6[6.1-6.9]$ & $>0.999^{c}$ \\
\hline General anaesthesia ${ }^{2}$ & $13(14)$ & $3(60)$ & $0.035^{a}$ \\
\hline Regional anaesthesia ${ }^{2}$ & $77(83)$ & $2(40)$ & $0.049^{a}$ \\
\hline Combined anaesthesia ${ }^{2}$ & $3(3)$ & $0(0)$ & $>0.999^{a}$ \\
\hline Procedure duration (hours) ${ }^{3}$ & $4.0[3.0-4.5]$ & $6.6[4.0-9.7]$ & $0.431^{c}$ \\
\hline Intraoperative RBC transfusion ${ }^{2}$ & $24(26)$ & $2(40)$ & $0.484^{a}$ \\
\hline Postop minimum haemoglobin $(\mathrm{g} / \mathrm{dl})^{1}$ & $10.1 \pm 1.5$ & $8.5 \pm 1.1$ & $0.042^{b}$ \\
\hline Postop minimum haematocrit (\%) ${ }^{1}$ & $30.5 \pm 5.0$ & $25.5 \pm 3.7$ & $0.031^{b}$ \\
\hline Postop maximum creatinine $(\mathrm{mg} / \mathrm{dl})^{3}$ & $1.0[0.9-1.5]$ & $2.3[1.9-6.1]$ & $0.002^{c}$ \\
\hline Postop maximum urea (mg/dl) $)^{3}$ & 34 [26-42] & $35[34-56]$ & $0.260^{c}$ \\
\hline Postoperative $\mathrm{AKI}^{2}$ & $11(14)$ & $4(80)$ & $0.003^{a}$ \\
\hline Postoperative RBC transfusion ${ }^{2}$ & $7(8)$ & $2(40)$ & $0.065^{c}$ \\
\hline ICU stay (days) ${ }^{3}$ & $1.0[1.0-2.0]$ & $11.0[8.5-29.5]$ & $<0.001^{c}$ \\
\hline Hospital stay (days) ${ }^{3}$ & $5.0[4.0-6.0]$ & $24[17.0-39.0]$ & $<0.001^{\mathrm{c}}$ \\
\hline
\end{tabular}

Table 1. Univariate analysis of predictors of MI.

RBC - red blood cell; Postop - postoperative

${ }^{1}$ mean $\pm \mathrm{SD} ;{ }^{2} \mathrm{n}(\%) ;{ }^{3}$ median [IQ25-75].

a Student's T test; b Chi-square test or Fisher test; c Mann-Whitney test. 